\title{
Design and Experimental Characterization of Hydraulically-actuated Revolute Joint based on Multimaterial Additive Manufacturing
}

\author{
A .Pfeil \\ ICube \\ INSA - CNRS - University of Strasbourg \\ antoine.pfeil@unistra.fr
}

\author{
T. P. Pusch \\ Fraunhofer IPA \\ Mannheim, Germany \\ tim.philipp.pusch@ipa.fraunhofer.de
}

\author{
F. Geiskopf \\ ICube \\ CNRS - University of Strasbourg \\ francois.geiskopf@insa-strasbourg.fr
}

\author{
M. Siegfarth \\ Fraunhofer IPA \\ Medical Faculty Mannheim \\ Mannheim, Germany \\ marius.siegfarth@ipa.fraunhofer.de
}

\author{
L. Barbé \\ ICube \\ CNRS - University of Strasbourg \\ laurent.barbe@unistra.fr

\section{P. Renaud* \\ ICube} \\ CNRS - University of Strasbourg \\ pierre.renaud@insa-strasbourg.fr
}

Design of fluidic actuators remain challenging in specific contexts such as the medical field, when solutions have for instance to be compatible with the stringent requirements of magnetic resonance imaging. In this paper, an innovative design of hydraulically-actuated revolute joint is introduced. The design originality is linked to the use of multimaterial additive manufacturing for its production. Hydraulic actuation and polymer manufacturing are selected to have compatibility with the medical context. A specific design taking advantage of the process capabilities is elaborated. The proposed component associates a large stroke compliant revolute joint and miniature pistons. An helical rack-and-pinion mechanism is integrated to the compliant joint to control the joint rotation. It is experimentally characterized in terms of range of motion, stiffness and available torque, to discuss the suitability of the component as a fluidic actuator. The component offers an interesting compactness, range of motion and the process is shown to be adequate for the design of functional systems.

\section{Introduction}

3D printing techniques have a clear impact on the design of robotic components and systems [1,2]. Multimaterial additive manufacturing (MMAM) opens even more design possibilities as demonstrated with its use for origami

*Address all correspondence to this author. structures [3], auxetic-based structures and actuators [4] or functionally graded materials in robotic structures [5].

Medical context is one of the application fields where MMAM seems particularly relevant. Robotic assistants often require compact architectures [6,7], and also compatibility with imaging devices [8]. For assistance in surgical tool positioning and guidance, safety requires in addition to limit the device dynamics and potentially introduce some compliance to help the robot-human interaction. These needs can be fulfilled with MMAM technologies and, among them, the Polyjet technology (Stratasys, USA) is commercially available. Polyjet process indeed allows the production of polymers which offer image compatibility $[9,10]$. Multimaterial components can help to reduce the number of parts in a system, so the cleaning of medical device is easier. Assembly can also be simplified and the freedom of shape helps to maximize compactness [11]. Soft materials can be printed [12], and Polyjet was also shown to endure gamma irradiation [9] for sterilization.

Hydraulics are well adapted to the actuation of 3D printed devices. In [13-15], hydraulic transmissions were efficiently used to perform actuation in a medical context. In terms of design, it is possible to benefit from the high power density of hydraulics and the possibility to have long transmission lengths to implement remote manipulation even in a challenging environment such as the MRI [16]. To our knowledge, there is however no component that was developed trying to benefit from the advantages of MMAM to de- 
sign hydraulically-driven robotic devices. This is our goal in this design innovation paper. We focused our attention on the elaboration of an actuated revolute joint to be used as an elementary component in medical robotic systems. The originality of the design comes from the early association in the design of the possibilities offered by hydraulic actuation and MMAM, namely the freedom of shape and material distribution. An active revolute joint is introduced, as it is the most common component in robotic systems. Initial work on this component was introduced in [17]. A proof of concept was then presented with a preliminary validation of kinematics. In this paper, the design is detailed, trying to exploit MMAM to maximize the component compactness. A complete characterization is performed so the interest of the design and future challenges in terms of design for additive manufacturing can be discussed.

In section 2, the actuated joint is introduced. The principle of operation is described before detailing the design of the component. In section 3, a thorough characterization is achieved. The behavior of the mechanical structure is investigated in terms of range of motion and stiffness. The whole component is then assessed to observe on one hand the influence of hydraulic actuation on the stiffness and on the other hand the final performance in terms of range of motion and static torque. Discussion and perspectives are then drawn in section 4 .

\section{The actuated joint design}

\subsection{Principle of operation}

The component is controlled using hydraulic power as an input, by means of pressurized hydraulic lines (Fig. 1). The output motion is a rotation, obtained using a simple architecture. It is composed of hydraulic cylinders to convert the hydraulic power into a mechanical power (1) and a rack and pinion mechanism (2) to transform the cylinder linear motion into a rotation, the output element being connected with a revolute joint to the ground (3). The rack and pinion mechanism was selected for the linearity of the translation/rotation transformation. Two single acting cylinders placed in tandem were preferred to a double acting cylinder to have a symmetrical arrangement in the component.

A CAD sectional view and prototype of the final component are introduced in Fig. 2. The active joint fits in a $40 \times 40 \times 30 \mathrm{~mm}^{3}$ volume and it offers \pm 60 degrees of amplitude. The specific implementation based on MMAM possibilities of the hydraulic cylinders and the revolute joint are detailed below.

\subsection{Design of hydraulic cylinders}

Polyjet technology enables the design of components composed of different polymer materials. The Young's modulus of materials ranges from $1 \mathrm{MPa}$ to $2000 \mathrm{MPa}$ [11], with respectively TangoBlack Plus and VeroWhite Plus materials. The associated Poisson ratios are equal to 0.49 and 0.3 , which means the soft material is nearly incompressible [12]. Any shape can be produced, but as the process is based on

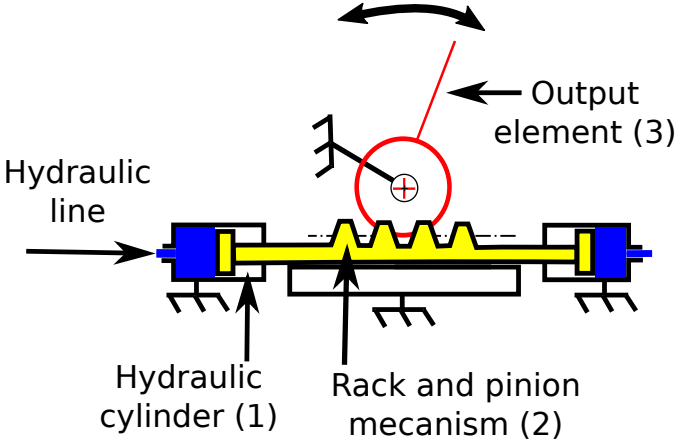

Fig. 1. Architecture of the active joint.
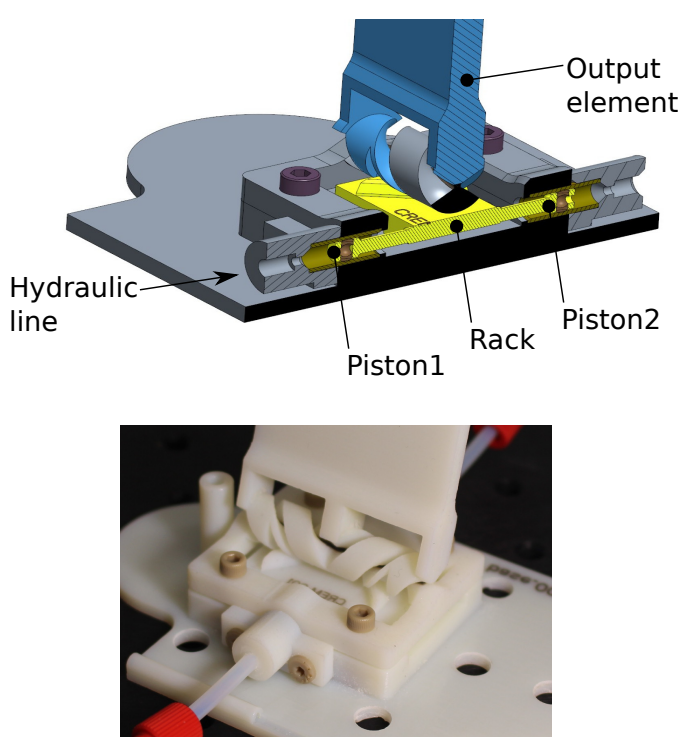

Fig. 2. CAD views of the actuated joint. (top) and rack-pinion integration(bottom)

the photopolymerization of jetted printed liquid polymer, a support material is needed during the production of hollow shapes or undercut surfaces.

The capacity to produce multimaterial components was exploited to design each piston as a single part, with a hollow shape at the tip. It was observed [18] this creates an adaptive sealing effect: friction between the piston and the bore is reduced for low fluid pressure, while high fluid pressure increases the friction to avoid leakage (Fig. ??). Risks of leakage between the seal and the piston rod are eliminated by merging the seal with the piston rod. With Polyjet process, the geometrical accuracy is about $0.1 \mathrm{~mm}$ [12], with shape defects of $0.1 \mathrm{~mm}$. The minimum thickness that can be printed consistently is $1 \mathrm{~mm}$, and the rugosity of surfaces is between 20 and 40 microns. The dimensions were chosen to minimize the piston size while taking into account these MMAM characteristics. It was observed it is possible to print reliably pistons of $4 \mathrm{~mm}$ diameter that are functional for about 100 cycles, which seems sufficient for use during a medical procedure, with a working pressure of 15 bars. The surface rugosity is not good enough to have a direct contact between printed surfaces of piston heads and cylinder bore. 


\begin{tabular}{|c|c|c|}
\hline Parameter & Designation & Value \\
\hline$d$ & Piston diameter & $4 \mathrm{~mm}$ \\
\hline$d_{0}$ & Joint external diameter & $10 \mathrm{~mm}$ \\
\hline$e$ & Soft material thickness & $1 \mathrm{~mm}$ \\
\hline$\alpha_{h s c}$ & Joint angle & $45^{\circ}$ \\
\hline
\end{tabular}

Table 1. Description of the component geometry
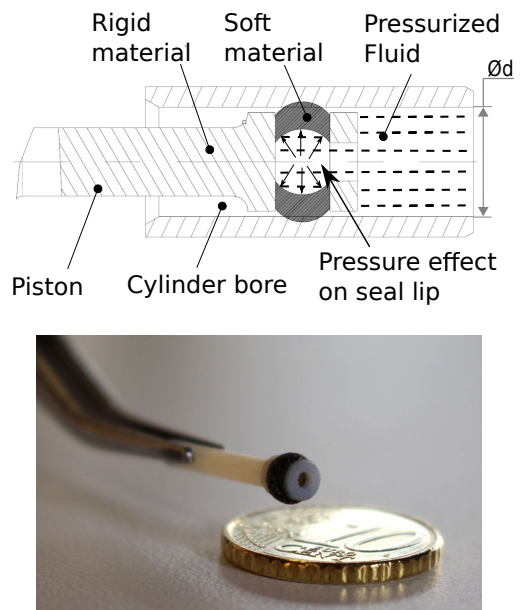

Fig. 3. Sectional view of the hydraulic cylinder (top) and piston prototype (bottom).

The final design of hydraulic cylinders is then composed of injection-molded bearings (H370SM- 0405-12, Igus, Germany) for the cylinder bore in contact with the MMAMbased pistons (see Fig. ??, Fig. 4 and Table 1 for detailed dimensions and designation of components).

\subsection{Design of the rack and pinion mechanism}

Wde used previously the freedom of shape and multimaterial capabilities to design of a compliant bimaterial revolute joint [12]. The joint shape is obtained by an helical sweep of the bimaterial notch-type joint profile represented in Fig. 4a. The compliant nature of the joint avoids the presence of dry friction. The joint stiffness is low along the rotation axis because of the use of a soft material at the center. Thanks to the helical sweep (Fig. 2), the stiffness is large in other directions as the incompressible soft material is submitted to compression for any loading direction. The admissible strain of the soft material makes it possible to have a range of motion in accordance with possible needs for medical robots, typically \pm 45 to \pm 90 degrees [8]. It was then selected as the base for the design of the actuated joint. The range of motion is set by the lateral surfaces (Fig. 4a), which is in addition interesting to offer safety by the simple restriction of range of motion.

The compliant joint profile is parametrized by an angle $\alpha_{h s c}$, a thickness $e$ and the external diameter $d_{0}$. The angle $\alpha_{h s c}$ sets the joint range of motion. It was set here to 60 degrees during the design, but it could be adjusted to respect other requirements. To minimize the component size, the parameters $e$ and $d_{0}$ are respectively set to $1 \mathrm{~mm}$ and $10 \mathrm{~mm}$ as these are the smallest possible values while respecting process specifications, according to [11]. For the same reason, the pitch of the helical sweep is chosen equal to $20 \mathrm{~mm}$. These parameters have then to be preserved during the rack and pinion integration, like the shape of lateral surfaces (Fig. 4a) that restrict the range of motion.

The approach we propose is to integrate the functional surface of the gear on the outer part of the joint cylindrical shape, as it is not used for kinematic guidance. In $[19,20]$, it was demonstrated that $3 \mathrm{D}$ printed gears can be obtained. But no method for dimensional synthesis of 3D printed rack and pinion mechanism was introduced to our knowledge. The design of the gear rack is rather simple as it can be integrated into the mechanical structure connecting the pistons. The gear rack is then designed as a separate component, obtained by merging the rack shape and the two multimaterial pistons. Conversely, the gear integration requires a specific method to minimize the component size by using the process freedom of shape. A normalized gear profile is defined by a pressure angle $\alpha_{0}$, a number of teeth $Z$ and a module $m$. A standard pressure angle of 20 degrees is considered. The gear profile needs to be adjusted to have the outer diameter equal to $d_{0}$. The gear can be manufactured only if all dimensions respect the minimum thickness of $1 \mathrm{~mm}$. We computed all possible geometries in terms of number of teeth and module. It appeared that there are no values of $m$ and $Z$ to have the gear fitting into the joint and that can be effectively printed.

The freedom of shape was then used to modify the gear section, considering the suppression of one tooth along the profile, as represented in Fig. 4c. The gear parameters are reported in Table 1. The actuated range of motion is \pm 45 degrees with a piston stroke of $6.3 \mathrm{~mm}$. In that case, it is possible to respect the constraints related to the manufacturing, without any modification of the lateral surfaces of the compliant joint. The gear overlap ratio is then equal to 1.25 , greater than 1 as desired for a rack and pinion mechanism [21].

Because of the helical shape of the compliant joint, the gear is also helical. The pitch of the helical sweep is related to the pitch diameter $d_{p}=m . Z$ and the gear helix angle $\beta$ with $\tan (\beta)=\pi . d_{p} /$ pitch so the helical angle $\beta=51.5^{\circ}$. The helix direction is reversed at the center of the compliant joint, in order to obtain a herringbone rack and pinion mechanism. This ensures that no axial force is generated on the compliant joint.

\section{Experimental characterization \\ 3.1 Characterization objectives}

The characterization of the actuated joint was achieved in two steps. First, the joint behavior was assessed when there is no fluid in the joint, by mechanically actuating the gear rack using a motorized linear stage. We designate this as the joint mechanical behavior. Then the overall component was studied when being actuated using hydraulics to get the component behavior. In this way, the impact of fluid behavior can be discussed. 


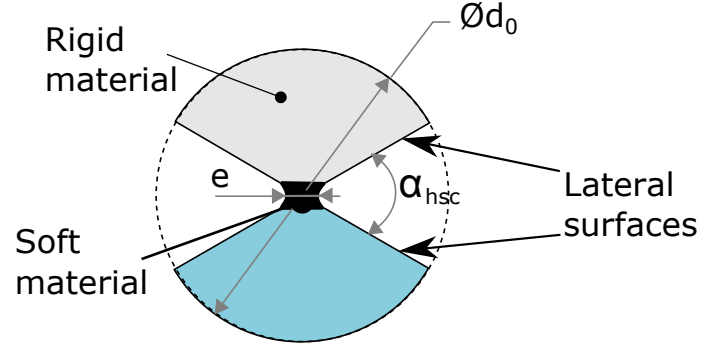

a)

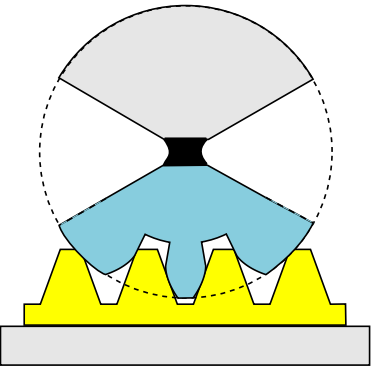

b)

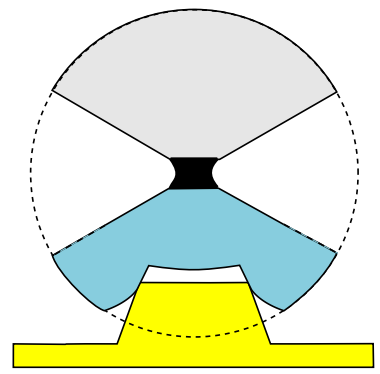

c)
Fig. 4. Description of compliant joint with integrated gear: a) Sectional view of the compliant joint (top), b) conventional gear integration (bottom left) and c) final design (bottom right)

The primary properties of the component we assessed are the range of motion and the torque that can be developed. Another important aspect is the impact of the use of a compliant joint. This latter introduces a compliance in translation $K_{x}$ and rotation $K_{\theta}$, as modeled in Figure 5. The hydraulic transmissions also necessarily introduce a compliance, that can be modeled as a stiffness $K_{t}$. In [11], the ratio between the translational $K_{x}$ and rotational compliance $K_{\theta}$ was measured and it showed there are no significant translational motions for the compliant joint alone. The compliance of the proposed active joint needs however to be assessed after the introduction of the rack and pinion mechanism and the hydraulic transmission.

As a consequence, the mechanical behavior is analysed in two phases. First the no-load input-output relationship is determined. Then a stiffness analysis is conducted. The evaluation of the component when using hydraulics is achieved by evaluating the range of motion, the developed torque, and again the stiffness.

\subsection{Mechanical behavior}

\subsubsection{No-load motion}

As a first step, the input-output relationship without external load is assessed with the setup on Figure 6. The rack is connected to a force sensor (K1107, Scaime, $20 \mathrm{~N}$ range, $0.01 \mathrm{~N}$ resolution), mounted on an actuated linear stage (FB075, Nanomotion, $100 \mathrm{~mm}$ range, $1 \mu \mathrm{m}$ resolution). The rack is displaced at a constant speed of $0.25 \mathrm{~mm} / \mathrm{s}$ along $5 \mathrm{~mm}$, which represents $80 \%$ of the total piston stroke to avoid over-constrain of the mechanism. The test was repeated three times in both directions. A telemeter (OptoNCDT 2320, Micro-epsilon, $0.03 \mu \mathrm{m}$ resolution) is positioned
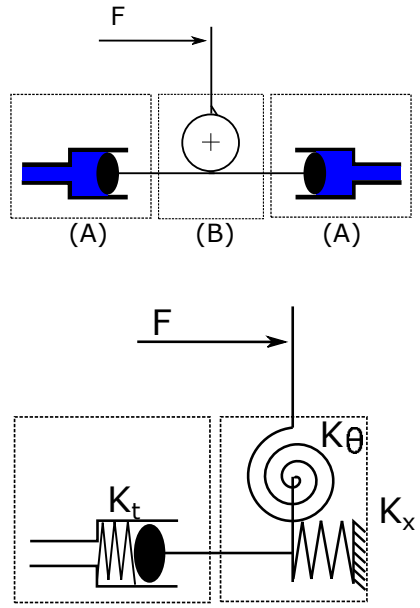

(A) (B)

Fig. 5. Modeling of the joint: Schematic representation (top) of the hydraulic lines $(A)$ with the joint $(B)$, the equivalent spring model (bottom).

at a height $h=8 \mathrm{~mm}$ above the axis of the rotational joint (Fig. 6) to measure the displacement $u$. A translation $t$ of the compliant joint may occur due to the force applied by the rack. It is neglected to compute the angular rotation $\theta_{z}$ as $\theta_{z}=\operatorname{Atan}(u / h)$. The observed relationship between the input translation $x$ and the output rotation $\theta_{z}$ is given on Figure 7 . The 3 cycles are superimposed on the figure, which shows the high level of repeatability of the joint motion. A $7^{\circ}$ hysteresis can be observed, which is the combination of the backlash in the experimental setup and in the rack-pinion mechanism. The rotation/translation ratio is experimentally evaluated equal to $13.6 \% \mathrm{~mm}$, which represents a $5 \%$ difference with the theoretical value, so the possible translation $t$ remains small. The maximum range of motion was then assessed by successive increases of the rack translation, until an increase of the force applied on the rack is denoted and considered as the presence of an internal mechanical contact. The range of motion is $79.6^{\circ}$, which represents a 11.5 $\%$ difference with the theoretical value of $90^{\circ}$. A possible explanation is the presence of unexpected contacts between the rack and the mechanical stops, compared to the nominal model, that could happen due to MMAM shape defects.

\subsubsection{Stiffness}

The stiffness was evaluated with the setup described in Figure 8. A force $F$ is exerted on the joint output with the same force sensor and linear stage, now placed at a distance $L=30 \mathrm{~mm}$ of the joint axis. The telemeter is aligned with the joint axis $(h=0 \mathrm{~mm})$. The piston is constrained mechanically. The compliant joint is submitted to the force $F$ and a moment $M_{z}=F$.L. From the position measurement with the telemeter and the linear stage, one can compute the translation $t$ and rotation $\theta_{z}$, and then determine the translational stiffness $K_{x}=F / t$ and the rotational stiffness $K_{\theta}=M_{z} / \theta_{z}$. Around the initial configuration, $K_{x}$ is equal to $21.4 \mathrm{~N} / \mathrm{mm}$. The torque-angle curve is given on Figure 9. The horizon- 

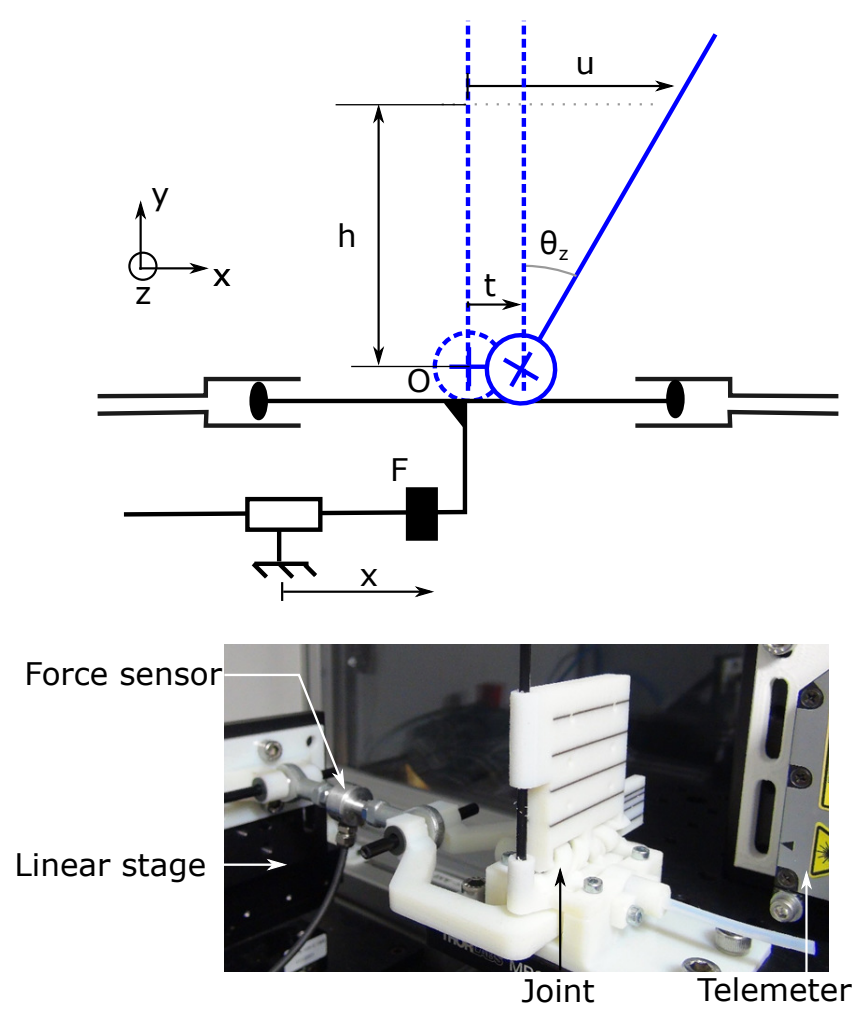

Fig. 6. Evaluation of input-output relationship: schematic view (top) and photograph of the set-up (bottom)

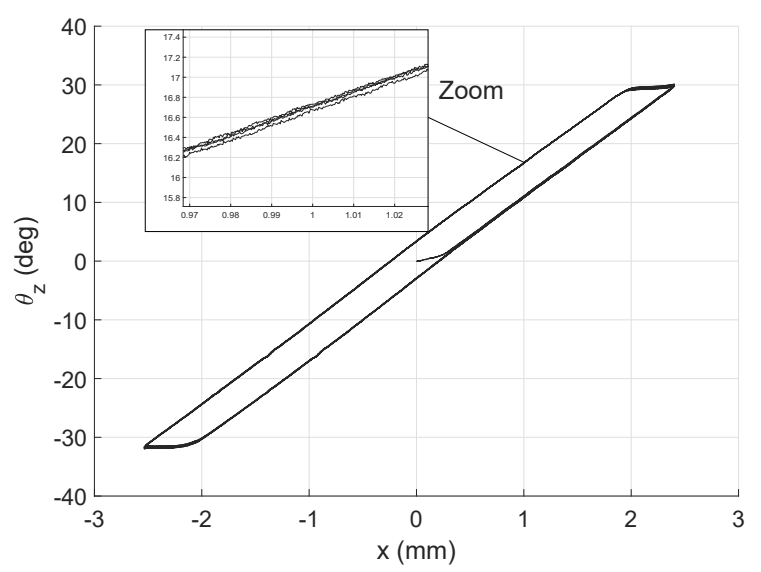

Fig. 7. Input-output relationship of joint in free motion without fluid.

tal domain corresponds to the joint backlash observed previously. Once the backlash is compensated, the mechanism has a stiffness equal to $K_{\theta}=4.8 \mathrm{Nmm} /{ }^{\circ}=275 \mathrm{Nmm} / \mathrm{rad}$.

One way to compare the stiffnesses $K_{x}$ and $K_{\theta}$ is to normalize them using a characteristic length $L_{R}$, as considered in [22,23]. With a length $L_{R}$ chosen equal to $50 \mathrm{~mm}$, the normalized translational stiffness is $K_{x}^{\prime}=K_{x} \cdot L_{R}^{2}=53500$ $\mathrm{Nmm} / \mathrm{rad}$, much higher than $K_{\theta}$. This means the application of a force mainly generates a rotation, and not a translation, so the mechanical component behaves like a compliant revolute joint.
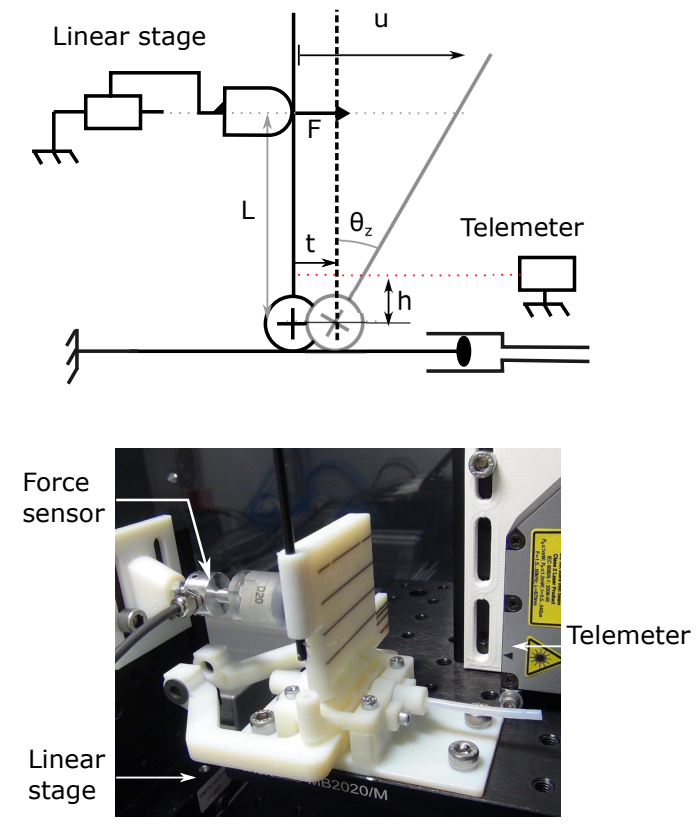

Fig. 8. Evaluation of stiffness: schematic view (top) and photograph of the set-up (bottom)

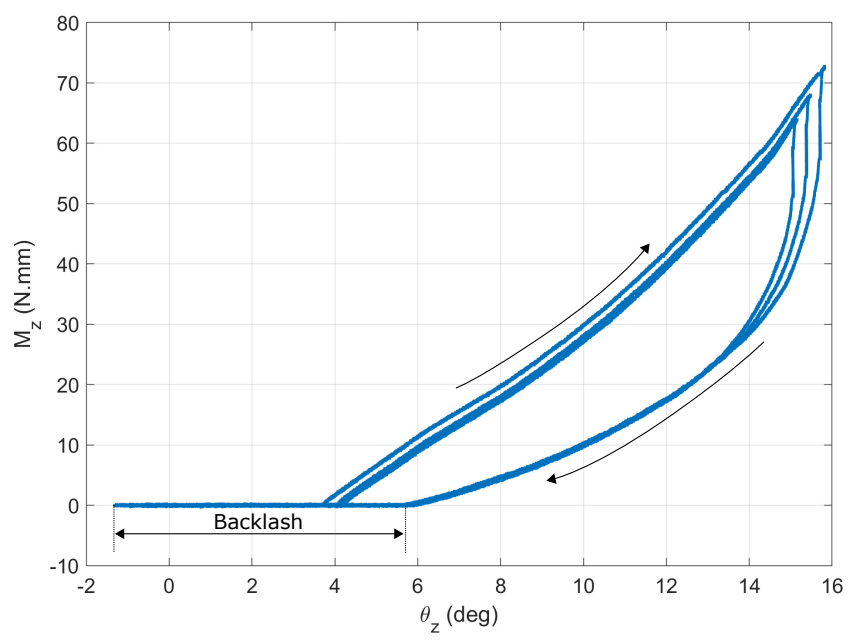

Fig. 9. Rotational stiffness: torque-angle curve.

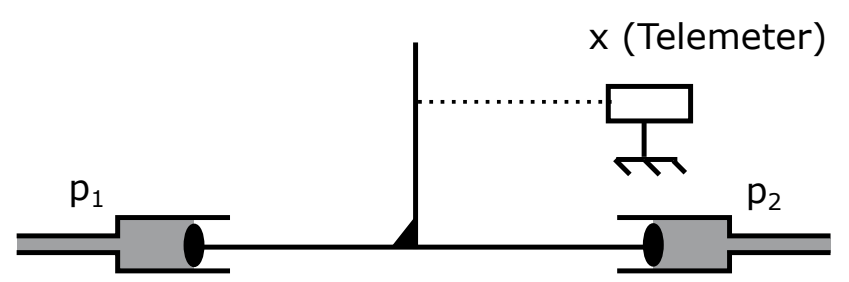

Fig. 10. Set-up for the characterization of pressure/velocity relationship with the hydraulic transmission.

\subsection{Component behavior}

\subsubsection{Evaluation of the hydraulic transmission}

To characterize the hydraulic transmissions, a simplified actuator without a revolute joint is used in order to directly measure linear movement of the hydraulic pistons. The motion is controlled by the application of a pressure differ- 


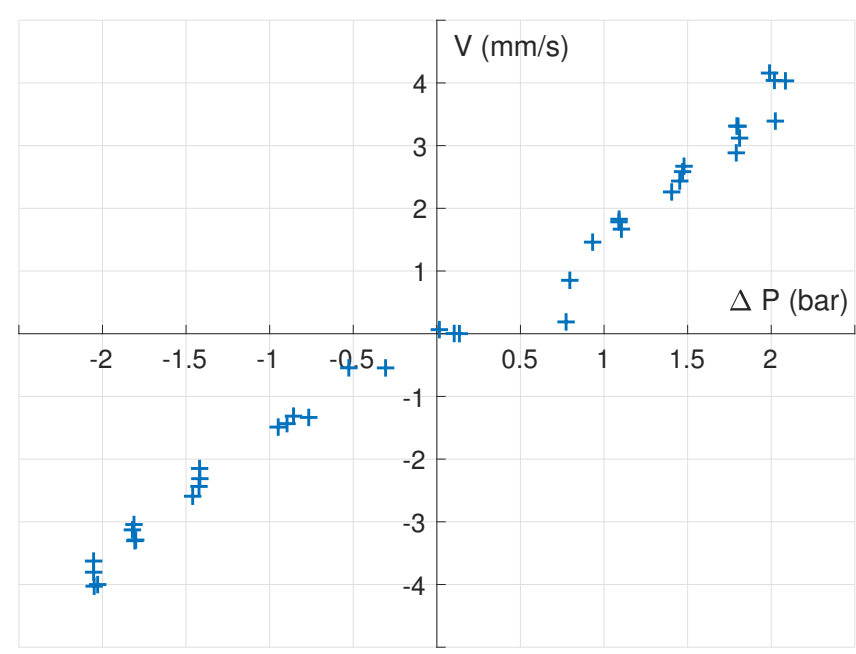

Fig. 11. Evolution of the relationship between velocity and pressure.

ence between the two hydraulic lines connected to the pistons. The pistons are connected to pressure-controlled syringe pumps (Nemesys, Cetoni, Germany) via $5 \mathrm{~m}$ PTFE tubes. It is then important to assess first the pressure/velocity relationship to evaluate the capability to control the component. The set-up is represented schematically in Figure 10. The figure 11 shows the measurement of the velocity $v$ as a function of the pressure difference $\Delta p$ between the two lines. Two quasi-linear domains can be identified for absolute pressure differences above 0.5 bar, and a dead band exists below this value, for velocities below $1 \mathrm{~mm} / \mathrm{s}$. Specific control would be needed for addressing motion control at such low speeds, which will be discussed in section 4 .

\subsubsection{Characterization of the component}

The range of motion is assessed with a setup similar to the one shown on Figure 6, the only difference being that the rack is moved by the hydraulic pressure instead of a linear stage. The pressure difference $\Delta p$ is initially set to 2 bars. Once the output rotation stops, $\Delta_{p}$ is set to -2 bars to get the reverse motion. The measured range of motion is $\left[-38.6^{\circ} ; 42.1^{\circ}\right]$, which yields a total range of $80.7^{\circ}, 10 \%$ lower than the theoretical range.

The static torque is assessed using the setup shown on figure 12. The torque is measured in the central position as schematically represented. The linear stage is locked in position while the pressure difference is increased. For $\Delta p=7$ bars, the static torque is reaching 30 N.mm.

The analysis of the mechanical behavior showed the main compliance of the component is in rotation. The rotational stiffness is thus assessed, using the setup shown on figure 12, with $h=0 \mathrm{~mm}$ for the distance between the linear stage and the joint axis. The pinion is initially placed and locked in the central position using an external mechanical lock. The hydraulic lines are pressurized up to a pressure $p_{0}$, after a degassing procedure to minimize the presence of air bubbles. The linear stage is then placed in contact with the component output, this contact being defined by a contact force of $0.1 \mathrm{~N}$ as measured with the force sensor. The linear

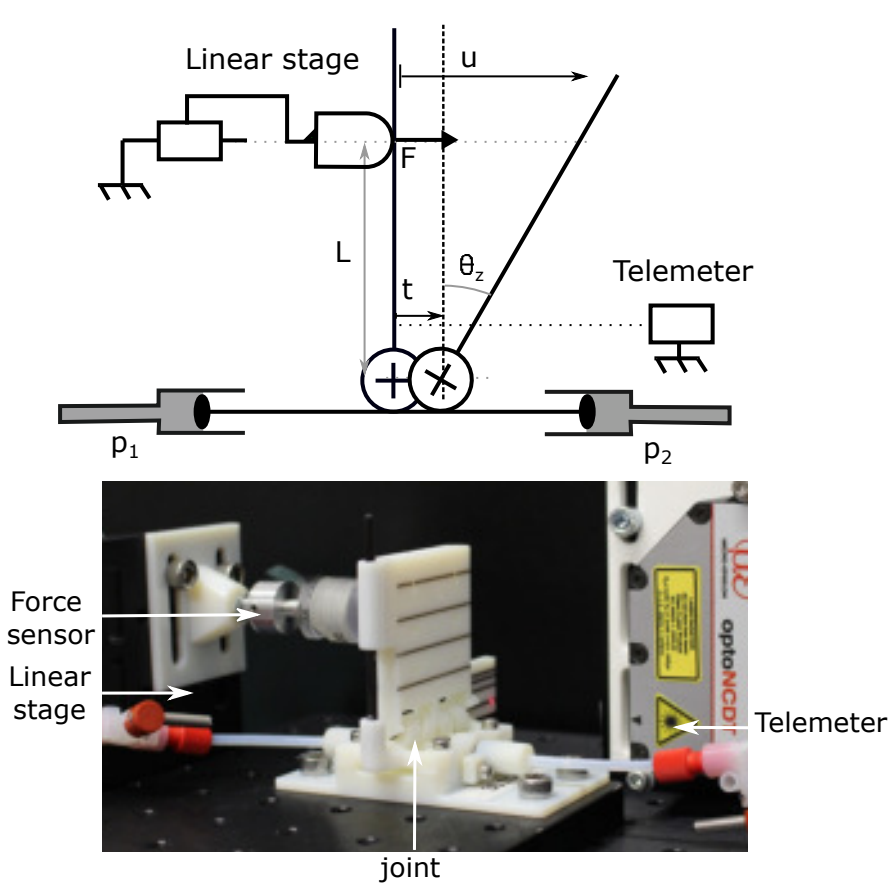

Fig. 12. Setup for characterization of torque and stiffness of the component: schematic view (top) and photograph (bottom).

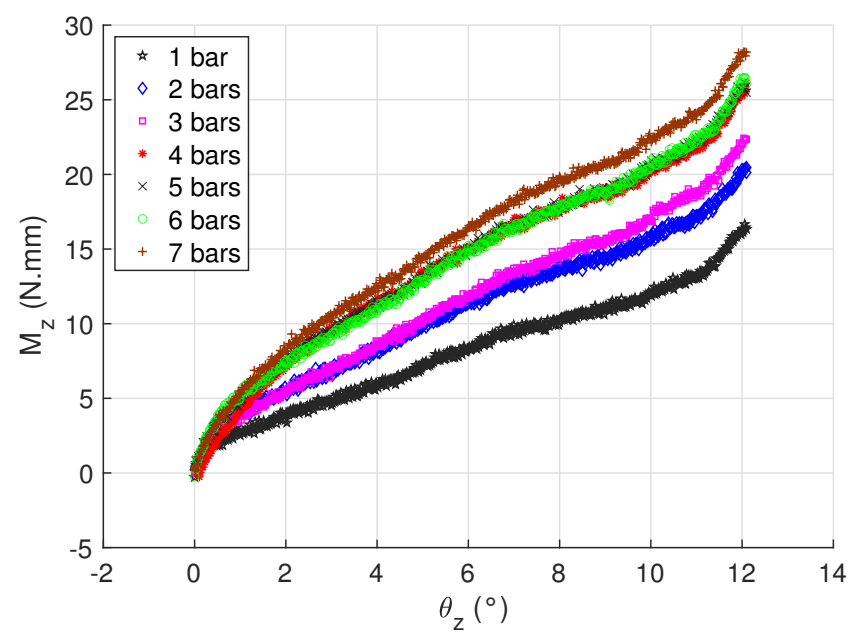

Fig. 13. Torque-angle curve as obtained during rotational stiffness assessment.

stage is stopped, and the mechanical lock is removed. Force and position of the linear stage are finally recorded while it is moved at a constant speed of $0.125 \mathrm{~mm} / \mathrm{s}$ along a displacement of $7.5 \mathrm{~mm}$, which corresponds to a pinion rotation of $12^{\circ}$. The moment $M_{z}$ and the angle $\theta_{z}$ are computed as described in section 3.2.

The relationship between $M_{z}$ and $\theta_{z}$ is plotted in figure 13. The rotational stiffness logically increases with the pressurization $p_{0}$. For rotations up to $5^{\circ}$, the average rotational stiffness at 1 bar is equal to $1.1 \mathrm{~N} . \mathrm{mm} /{ }^{\circ}$, while at 7 bars, it is doubled at $2.6 \mathrm{~N} \cdot \mathrm{mm} /{ }^{\circ}$. 


\section{Discussion and conclusion}

The prototype offers a range of motion which is $90 \%$ of the design value. With the geometry we produced, the effective range is over $80^{\circ}$ which corresponds to the needs described in the introduction for the medical context. If another value is needed, it is simple to redesign the component by changing the angle $\alpha_{h s c}$ (Fig. 4) and the stroke of the hydraulic cylinders. The discrepancy between the nominal and effective motion amplitudes seems to come from early contact between the helical shapes in the compliant joint. The impact of MMAM production could be compensated by shifting the value of $\alpha_{h s c}$ before production as tested in [11]. In parallel, an increase of the torque capacity could be obtained if needed by adjusting the piston diameter and the diameter $d_{0}$ of the compliant revolute joint as it integrates the gear.

The joint stiffness is significantly impacted by the stiffness of the hydraulic lines. The rotational stiffness changes from $4.8 \mathrm{Nmm} /{ }^{\circ}$ down to $1.9 \mathrm{Nmm} /{ }^{\circ}$. This value is obtained with significant transmission lengths, since the tubes are $5 \mathrm{~m}$ long to reflect a possible use for remote manipulation in MRI environment. This noticeable impact of hydraulic lines can be seen as a limit for tasks requiring very high accuracy. On the opposite, the use of hydraulics provides naturally some compliance that can be beneficial for control during humanrobot interaction.

The use of MMAM allowed us to build an actuated hydraulic joint of quite small size, with a simple kinematic architecture and a limited number of components. Thanks to the integration of soft and rigid materials, the rack and the pistons are built as one part. Similarly, the gear and the revolute joint are merged in a one part. Without the freedom of shape of MMAM, it would be very difficult to envision such geometries. Our evaluation shows we are able to use pressures up to 7 bars, with a lifetime that is according to our experience of several tens of cycles. Designing an MMAMbased hydraulic joint can then help to build compact custom systems for single use or limited number of uses, as often considered in the medical field. In terms of compactness, this solution is here interesting when compared to designs based on rolling diaphragms $[13,15]$, as these diaphragms are limited in terms of stroke for a given diameter. Another advantage of the proposed component is to ease the design of devices with specific kinematic architectures, for instance with spatial linkages as considered in parallel mechanisms, as the relative position of rotation axes is ensured by manufacturing.

There are still limits to the proposed hydraulic-based actuated joint in its current implementation. First, the use of Polyjet process as considered here with TangoBlack plus material was observed to suffer from material degradation for long-term uses [18]. Further process development would help to have functional systems with large endurance. Even though the revolute joint is compliant, the actuated joint still demonstrates some backlash due to the inaccuracies between the rack and the pinion. Process improvement in terms of resolution and accuracy would help to improve the component performance. There is a dead band in the pressure/velocity relationship that would require advanced control strategies if one needs to have high-resolution motion control. Solutions based on rolling diaphragms have here a clear advantage, thanks to the absence of dry friction. In other words, the proposed hydraulically-actuated revolute joint can be seen as a component with performances that are complementary to other solutions. As of today, it can be a design innovation to develop new robotic devices, and it will benefit from progress in in the field of MMAM. One ongoing perspective of this work is to assess a complete robotic system based on such a joint. Another perspective is to determine how MMAM can be exploited to build other basic components such as an actuated prismatic joint for the same type of applications. On a long term, it would be of great interest to develop methodologies in order to help the design for additive manufacturing. It would be very helpful for instance to help the designer to identify best decomposition of a robotic system into multi-material sub-components, exploiting the freedom of material distribution and shape while respecting the process limits in terms of part cleaning, production time and production volume.

\section{Acknowledgements}

This work was supported by the INTERREG Upper Rhine program from the ERDF (European Regional Development Fund), SPIRITS project, as well as the Investissements d'Avenir (Robotex ANR-10-EQPX-44, Labex CAMI ANR-11- LABX-0004).

\section{References}

[1] Morrow, J., Hemleben, S., and Menguc, Y., 2017. "Directly Fabricating Soft Robotic Actuators With an Open-Source 3-D Printer". IEEE Robotics and Automation Letters, 2(1), Jan., pp. 277-281.

[2] Mehta, A., DelPreto, J., and Rus, D., 2015. "Integrated Codesign of Printable Robots". Journal of Mechanisms and Robotics, 7(2), May, p. 021015.

[3] Liu, T., Wang, Y., and Lee, K., 2018. "ThreeDimensional Printable Origami Twisted Tower: Design, Fabrication, and Robot Embodiment". IEEE Robotics and Automation Letters, 3(1), Jan., pp. 116123.

[4] Schmitt, F., Piccin, O., Bayle, B., Renaud, P., and Barbé, L., 2020. "Inverted Honeycomb Cell as a Reinforcement Structure for Building Soft Pneumatic Linear Actuators". Journal of Mechanisms and Robotics, 13(1), 11.

[5] Bartlett, N. W., Tolley, M. T., Overvelde, J. T. B., Weaver, J. C., Mosadegh, B., Bertoldi, K., Whitesides, G. M., and Wood, R. J., 2015. "A 3d-printed, functionally graded soft robot powered by combustion". Science, 349(6244), pp. 161-165.

[6] Elhawary, H., Tse, Z. T. H., Hamed, A., Rea, M., Davies, B. L., and Lamperth, M. U., 2008. "The case for MR-compatible robotics: a review of the state of 
the art". The International Journal of Medical Robotics and Computer Assisted Surgery, 4(2), pp. 105-113.

[7] Comber, D. B., Slightam, J. E., Gervasi, V. R., Neimat, J. S., and Barth, E. J., 2016. "Design, Additive Manufacture, and Control of a Pneumatic MR-Compatible Needle Driver". IEEE Transactions on Robotics, 32(1), Feb., pp. 138-149.

[8] Pfeil, A., Barbé, L., Geiskopf, F., Cazzato, R. L., and Renaud, P., 2021. "Workflow-Based Design and Evaluation of a Device for CBCT-Guided Biopsy". Journal of Medical Devices, 03.

[9] Pfeil, A., Barbé, L., Wach, B., Bruyas, A., Geiskopf, F., Nierenberger, M., and Renaud, P., 2018. "A 3D-printed needle driver based on auxetic structure and inchworm kinematics". In ASME 2018 International Design Engineering Technical Conferences.

[10] Neumann, W., Pusch, T. P., Siegfarth, M., Schad, L. R., and Stallkamp, J. L., 2019. "CT and MRI compatibility of flexible 3d-printed materials for soft actuators and robots used in image-guided interventions". Medical Physics, 46(12), pp. 5488-5498.

[11] Bruyas, A., Geiskopf, F., and Renaud, P., 2015. "Design and Modeling of a Large Amplitude Compliant Revolute Joint: The Helical Shape Compliant Joint”. Journal of Mechanical Design, 137(8), Aug., pp. 085003-085003-8.

[12] Bruyas, A., Geiskopf, F., Meylheuc, L., and Renaud, P., 2014. "Combining Multi-Material Rapid Prototyping and Pseudo-Rigid Body Modeling for a new compliant mechanism". In IEEE International Conference on Robotics and Automation (ICRA), pp. 3390-3396.

[13] Dong, Z., Guo, Z., Lee, K., Fang, G., Tang, W. L., Chang, H., Chan, D. T. M., and Kwok, K., 2019. "High-performance continuous hydraulic motor for $\mathrm{mr}$ safe robotic teleoperation". IEEE Robotics and Automation Letters, 4(2), pp. 1964-1971.

[14] Whitney, J. P., Glisson, M. F., Brockmeyer, E. L., and Hodgins, J. K., 2014. "A low-friction passive fluid transmission and fluid-tendon soft actuator". In 2014 IEEE/RSJ International Conference on Intelligent Robots and Systems, pp. 2801-2808.

[15] Burkhard, N., Frishman, S., Gruebele, A., Whitney, J. P., Goldman, R., Daniel, B., and Cutkosky, M., 2017. "A rolling-diaphragm hydrostatic transmission for remote MR-guided needle insertion”. In IEEE International Conference on Robotics and Automation (ICRA), pp. 1148-1153.

[16] Ganesh, G., Gassert, R., Burdet, E., and Bleuler, H., 2004. "Dynamics and control of an MRI compatible master-slave system with hydrostatic transmission". In IEEE International Conference on Robotics and $\mathrm{Au}-$ tomation, 2004. Proceedings. ICRA '04. 2004, IEEE, pp. 1288-1294 Vol.2.

[17] Pfeil, A., Siegfarth, M., Geiskopf, F., Pusch, T., Barbé, L., and Renaud, P., 2019. "Hydraulically-actuated compliant revolute joint for medical robotic systems based on multimaterial additive manufacturing”. In IEEE International Conference on Robotics and Automation,
2019. Proceedings, Vol. 2, IEEE, pp. 8401-8407.

[18] Siegfarth, M., Pusch, T., Pfeil, A., and Stallkamp, J., 2020. "Multi-material 3d printed hydraulic actuator for medical robots". Rapid Prototyping Journal, 26(6), pp. 1019-1026.

[19] Morimoto, T., Hawkes, E., and Okamura, A., 2017. "Design of a compact acutation and control syste for flexible medical robots". IEEE Robotics and Automation Letters, 2(3), pp. 1579-1585.

[20] Groenhuis, V., Siepel, F., Veltman, J., and Stramigioli, S., 2017. "Design and characterization of stormram 4: an MRI-compatible robotic system fro breast biopsy". In IEEE/RSJ International Conference on Intelligent Robots and Systems (IROS), pp. 928-933.

[21] Radzevich, S. P., 2012. Dudley's Handbook of Practical Gear Design and Manufacture, Second Edition. CRC Press, Apr.

[22] Moon, Y., Trease, B. P., and Kota, S., 2002. "Design of large-displacement compliant joints". Proceedings of ASME IDETC 2002, p. 34207.

[23] Trease, B. P., Moon, Y., and Kota, S., 2005. "Design of large-displacement compliant joints". Journal of Mechanical Design, 127(4), pp. 788-798. 